\title{
Traceable dynamic measurement of mechanical quantities: objectives and first results of this european project
}

\author{
C. Bartoli ${ }^{1, \star}$, M.F. Beug ${ }^{2}$, T. Bruns ${ }^{2}$, C. Elster ${ }^{3}$, T. Esward ${ }^{4}$, L. Klaus ${ }^{2}$, A. Knott ${ }^{4}$, M. Kobusch ${ }^{2}$, \\ S. Saxholm ${ }^{5}$, and C. Schlegel ${ }^{2}$ \\ 1 LNE, 1 rue Gaston Boissier, 75015 Paris, France \\ 2 PTB, Bundesallee 100, 38116 Braunschweig, Germany \\ 3 PTB, Abbestraße 2-12, 10587 Berlin-Charlottenburg, Germany \\ 4 NPL, Hampton Road, TW11 0LW Teddington, UK \\ 5 MIKES, Tekniikantie 1, 02150 Espoo, Finland
}

Received: 12 October 2012 / Accepted: 28 October 2012

\begin{abstract}
Nine european national metrology institutes (NMIs) are collaborating in a new project funded by the european metrology research programme (EMRP) to establish traceable dynamic measurement of the mechanical quantities force, pressure, and torque. The aim of this joint research project (JRP) is to develop appropriate calibration methods, mathematical models, and uncertainty evaluation. The duration of the project is 3 years for a global amount of $€ 3.6$ million. It began in September 2011.
\end{abstract}

Keywords: Dynamic force; dynamic torque; dynamic pressure; traceability; measurement uncertainty

\section{Introduction}

In aerospace, medicine, production, transport and process control, dynamic measurements are generally performed under dynamic conditions, whereas sensors and measuring amplifiers are typically calibrated under static conditions. High-speed data acquisition and modelling are necessary to develop advanced dynamic measurement methods. With important applications in the automotive industry, engineering, petrochemical and pharmaceutical industries, but also for the study of properties of materials, the development of new technologies and standards is required.

The project directly addresses the current lack of traceability for the measurement of dynamic mechanical quantities, including traceability of the response of transducers and of signal acquisition and conditioning instrumentation to dynamic stimuli. Achieving traceability also requires new developments in modelling and uncertainty analysis and propagation. The next generation of metrology systems will rely on further advances in mathematics and information technology, and on the development of reliable mathematical models, enhanced capabilities for data analysis, and trustworthy software [1].

The project is split into five technical work packages that address the following topics:

- dynamic force (WP 1);

- dynamic pressure (WP 2);

* Correspondence: claire.bartoli@lne.fr

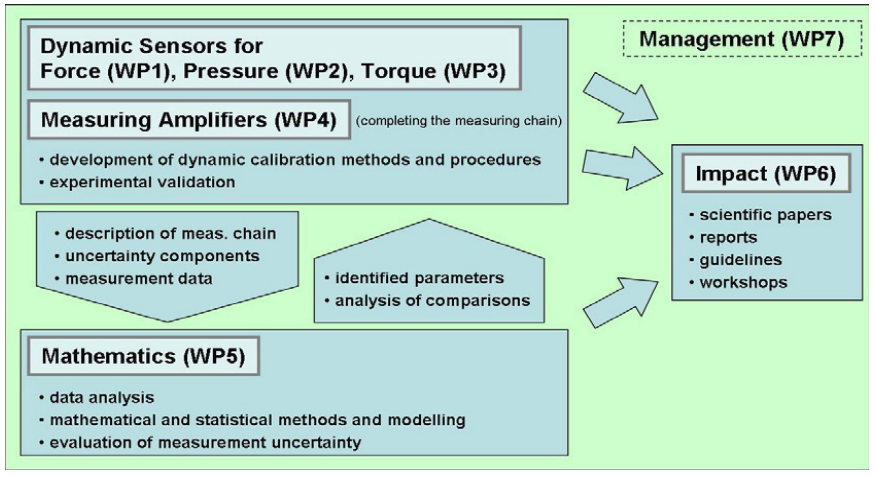

Fig. 1. Coordination and interaction between work packages.

- dynamic torque (WP 3);

- characterisation of measuring amplifiers (WP 4);

- mathematical and statistical methods and models (WP 5).

The coordination and the interaction between the different work packages is described in Figure 1.

The subsequent sections of this paper describe the aims and recent achievements of each technical work package.

\section{WP 1: dynamic force}

This work package aims to provide methods and standards for the measurement of sinusoidal and shock forces. 
Table 1. List of chosen force transducers.

\begin{tabular}{lccc}
\hline & Transducer-1 & Transducer-2 & Transducer-3 \\
\hline Type & Kistler 9175B & HBM U9B & Interface 1610 \\
Range & $8 \mathrm{kN} / \pm 4 \mathrm{kN}$ & $\pm 1 \mathrm{kN} / \pm 1 \mathrm{kN}$ & $\pm 2 \mathrm{kN} / \pm 2 \mathrm{kN}$ \\
Frequency & $10 \mathrm{~Hz}-1.5 \mathrm{kHz}$ & $10 \mathrm{~Hz}-1.5 \mathrm{kHz}$ & $10 \mathrm{~Hz}-1.5 \mathrm{kHz}$ \\
Amplifier & Charge & Bridge & Bridge \\
& amplifier & amplifier & amplifier \\
\hline
\end{tabular}

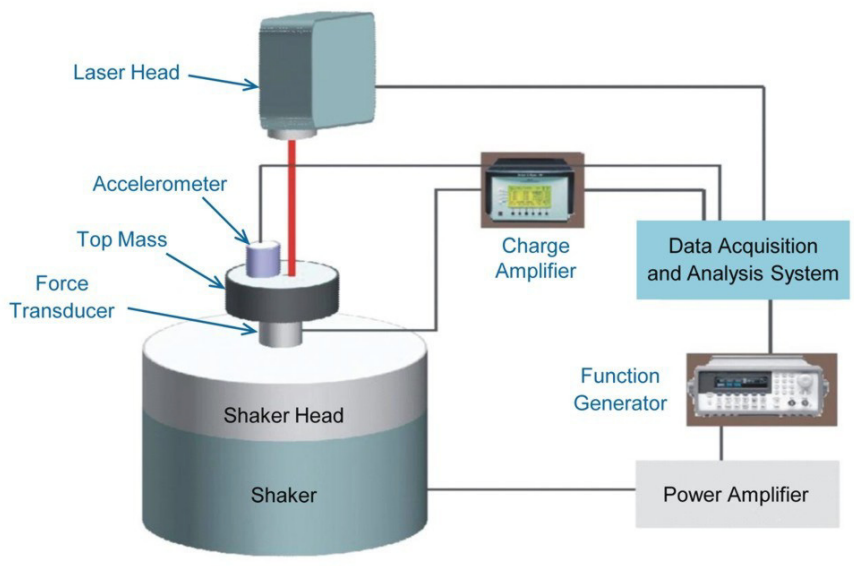

Fig. 2. Schematic of the measurement set-up.

Primary sine force facilities will cover the frequency domain from DC to $2 \mathrm{kHz}$ with amplitudes up to $10 \mathrm{kN}$. For shock domain calibration, amplitudes up to $250 \mathrm{kN}$ will be possible.

\subsection{Sinusoidal force calibration facilities}

Participants are CEM (Spain), LNE (France) and PTB, who is the pilot.

A set of force transducers to be used in the scope of this project was defined and is presented in Table 1 .

\subsubsection{Principle of measurement}

The three participants have developed their own facilities, which all work according to the principle described below.

Each shaker system consists of an electrodynamic exciter and an appropriate power amplifier. A function generator supplies the desired excitation signal. This signal directly modulates the current that drives the coil of the shaker armature. The acceleration of the top mass connected to the force transducer under test defines the traceability of the acting dynamic force. This acceleration is measured by means of a Polytec laser vibrometer or by a reference accelerometer. The principal measurement setup for sinusoidal force excitation is shown in Figure 2. Detailed information about the measuring principle and the evaluation methods for sinusoidal force calibration is found in $[2,3]$.

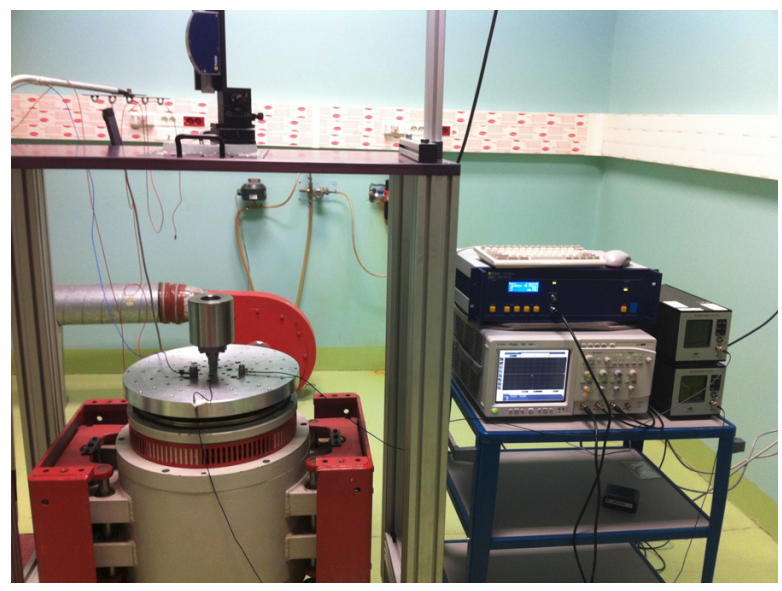

Fig. 3. Photo of the CEM installation.

\subsubsection{CEM and LNE facilities}

The CEM facilities for dynamic force measurement include the followings parts and are shown in Figure 3:

- Shaker LDS 726 with amplifier PA 2000.

- Laser vibrometer Polytec CLV 2534.

- Oscilloscope Agilent Infinium DSO8064A.

- Force transducer Kistler Type 9175 B.

- Single ended accelerometer B\&K 8305.

- Two signal conditioners B\&K 2525, to be used with force sensor and accelerometer.

The LNE facilities include the followings parts:

- Shaker LDS 721 with amplifier PA 2000.

- Data Physics Mobilizer acquisition and generator system.

- Force transducer Kistler Type 9175 B.

- Single ended accelerometer B\&K 8305.

- Two signal conditioners B\&K 2525 and 2626, to be used with force sensor and accelerometer.

\subsubsection{PTB facilities}

At PTB three electrodynamic shaker systems are available for the sinusoidal dynamic force calibrations to be made within this JRP. Their specifications are as follows:

- B\&K-Shaker, force amplitude up to $100 \mathrm{~N}$, frequency range $10 \mathrm{~Hz}$ to $2 \mathrm{kHz}$.

- R\&S-Shaker, up to $800 \mathrm{~N}, 10 \mathrm{~Hz}$ to $3 \mathrm{kHz}$, mounted in a rigid frame.

- LDS-Shaker, up to $10 \mathrm{kN}, 10 \mathrm{~Hz}$ to $2 \mathrm{kHz}$ (cf. Fig. 4).

In the first month of the project, the infrastructure has been improved and experimental investigations with single-point and scanning laser vibrometers by Polytec were carried out at PTB.

The acceleration of the top mass is measured by a laser method. Two different vibrometers are now available:

- a single-point vibrometer with a fixed measuring beam; - a scanning vibrometer that scans the target surface. 


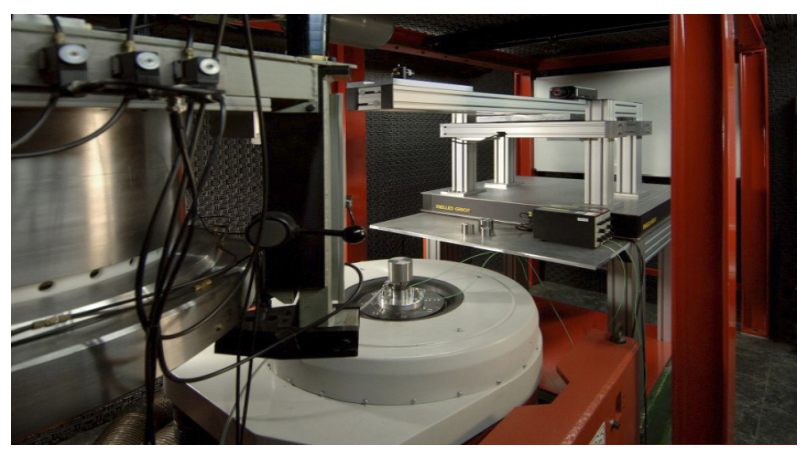

Fig. 4. Photo of the PTB installation.

The scanning vibrometer is able to scan the acceleration distribution of the surface within an angle range of $\pm 25^{\circ}$ in $x / y$ direction ( $z$ direction is defined as the vibration axis). Its laser head features an integrated digital camera system as well as a $3 \mathrm{D}$ unit to determine the spatial coordinates of the measuring points. The alignment of the camera system and the laser beam is realized by a special optical unit, the so-called coaxial unit.

The modular vibrometer controller is equipped with different digital processing units, two velocity decoders, a displacement decoder and a digital quadrature decoder. The controller provides a frequency range from $0 \mathrm{~Hz}$ to $2.5 \mathrm{MHz}$ with a maximum velocity of $10 \mathrm{~m} / \mathrm{s}$.

The digital quadrature encoder is used with the software VibSoft from Polytec that calculates the displacements according to the arc tangent procedure. The analogue $I / Q$ output signals, the transducer output signal and the acceleration signal from the shaker armature measured by another accelerometer fixed on the shaker table are bundled in a junction box feeding a $\mathrm{PC}$ with $5 \mathrm{MHz}$ sampling card from National Instruments.

\subsubsection{Resonance of a piezoelectric force transducer}

The resonance behaviour of a piezoelectric force transducer 9175B from Kistler with connected top mass was investigated on the LDS shaker system applying the above described laser vibrometers.

Three measurement signals were acquired: the acceleration of the top mass, the force signal, and the acceleration of the shaker table. The shaker was excited with a periodic-chirp signal. The FFT analysis of the time data yields the frequency response of the acceleration ratio between top mass and shaker table. The two plots shown in Figure 5 present the amplitude and phase response of the acceleration ratio of top mass and shaker table as a function of the excitation frequency.

By model-based fitting of the measured frequency response it is possible to identify the dynamic parameters (i.e., stiffness, damping) of the force transducer.

\subsection{Shock force calibration facilities}

Force calibrations with pulse-shaped forces will be performed on two devices specified for $20 \mathrm{kN}$ and $250 \mathrm{kN}$ shock force amplitude at PTB [4-6]. Both devices use two

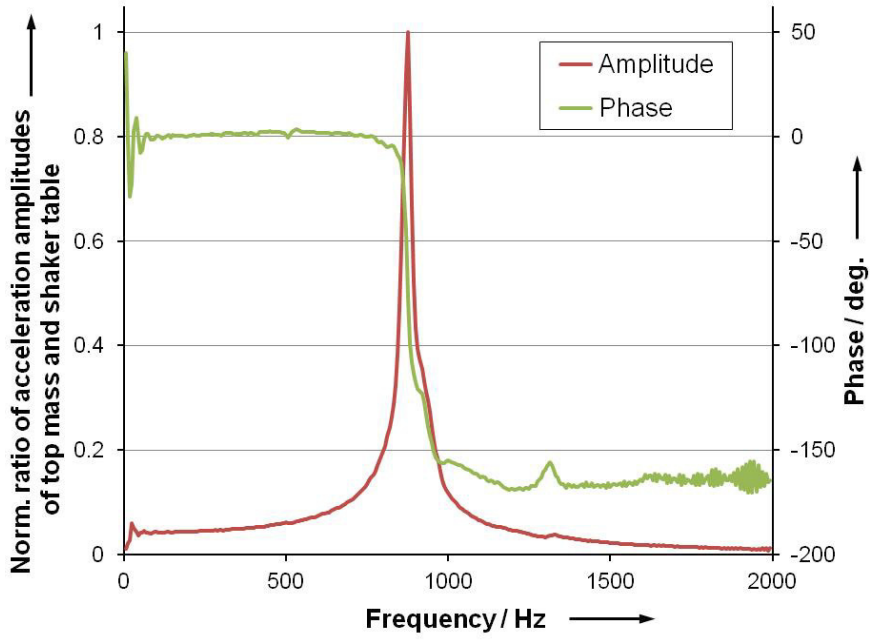

Fig. 5. Measured resonance behaviour of a piezoelectric force transducer with a top mass of $6 \mathrm{~kg}$.

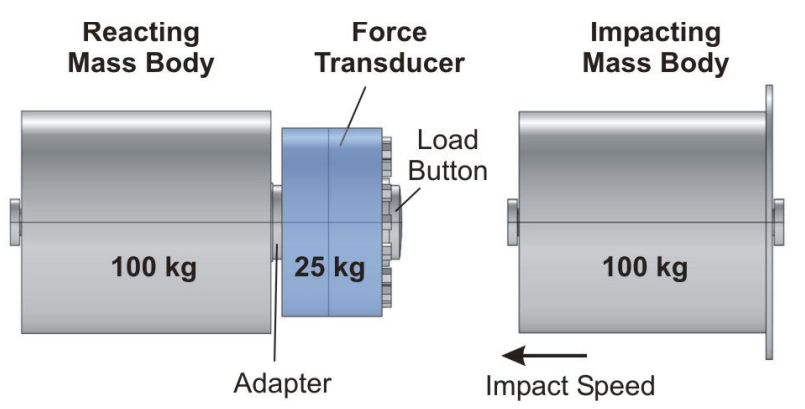

Fig. 6. Drawing of the impact configuration of the $250 \mathrm{kN}$ shock force calibration device with a heavy force transducer.

colliding impact mass bodies guided by air bearings. Measurements of the inertial forces during the moment of impact by laser vibrometers provide traceability of the dynamic force.

Within the scope of this EMRP project, the $20 \mathrm{kN}$ shock force calibration device was modified to improve the signal quality of the vibrometer measurement by applying an on-axis measurement geometry, a geometry which was already used for the larger $250 \mathrm{kN}$ device. For this purpose, some mechanical modifications of the acceleration mechanism and the shock damper were made to match with a new mounting arrangement of the vibrometers mounted on active vibration isolation tables.

For the development of methods for validated primary shock force calibration, a model-based analysis of the dynamic behavior of the $250 \mathrm{kN}$ shock force calibration device was carried out to understand the modal oscillations that could be observed for heavy force transducers under test [7]. Figure 6 sketches the analysed geometrical impact configuration consisting of the two cylindrical impact mass bodies (about $100 \mathrm{~kg}$ each) and the adapted force transducer under test (about $25 \mathrm{~kg}$ ).

A typical measurement result of a hard metallic impact shock is shown in Figure 7. The diagram displays the signals of the force transducer (black line) and the two inertial forces (red lines) calculated from the accelerations 


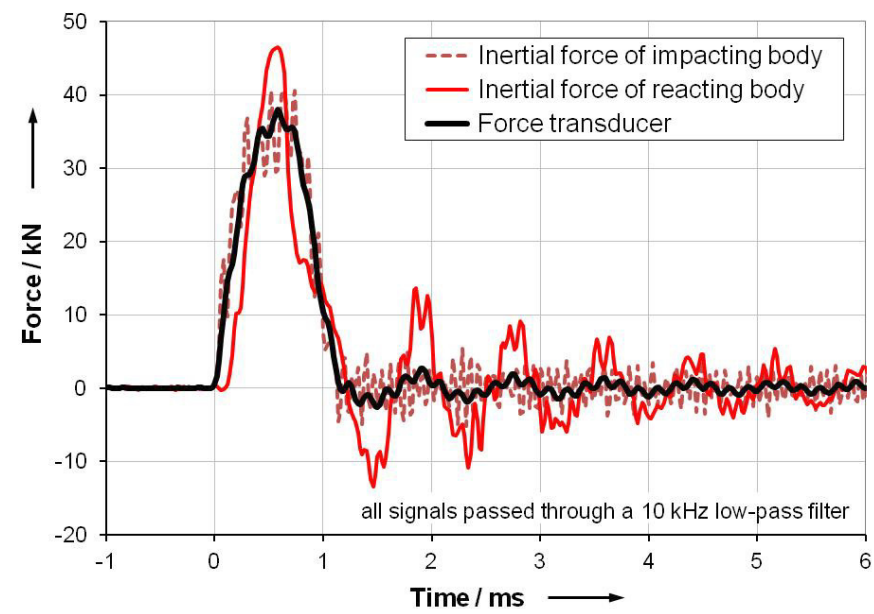

Fig. 7. Shock force measurement with a heavy force transducer showing modal oscillations.

measured by the vibrometers at both opposing on-axis positions. With a pulse width of about $1.1 \mathrm{~ms}$, the shock excited several modal oscillations which were identified by the application of auxiliary acceleration sensors and by means of a finite element analysis of the mechanical impact configuration.

\section{WP 2: dynamic pressure}

This work package is concerned with development of primary dynamic pressure standards and secondary systems to calibrate industrial transducers.

The work package participants are SP (Sweden), MIKES (Finland) and PTB under the leadership of NPL.

\subsection{Drop weight machines}

The dynamic pressure facilities of MIKES and PTB operate according to the "drop weight" principle. At PTB, a rigid mass ball of about $3 \mathrm{~kg}$ is dropped on a piston of a cross sectional diameter of approximately $8 \mathrm{~mm}$ [8]. At MIKES, the principle is the same, but the cylindrical mass body is about $14 \mathrm{~kg}$ and the piston diameter is of the order of a few centimeters.

The following figures show the design of the two machines at MIKES (cf. Fig. 8) and PTB (cf. Fig. 9).

The impact on the piston leads to the compression of a small volume of a hydraulic liquid within a pressure cavity that is connected to the device(s) under test (DUT), thus applying a shock pressure excitation to the DUT.

Commonly these devices are used for the comparison of DUT(s) with a reference transducer in a secondary calibration scheme. However, within the JRP, the laboratories follow two different approaches to obtain a direct pressure measurement without a reference sensor.

Figure 10 presents a dynamic pressure measurement (unfiltered data) performed at MIKES showing the deceleration of the drop weight and the corresponding pressure pulse of about $2.5 \mathrm{~ms}$ pulse length.

The MIKES device is modified to measure the motion of the piston or the deceleration of the drop-weight

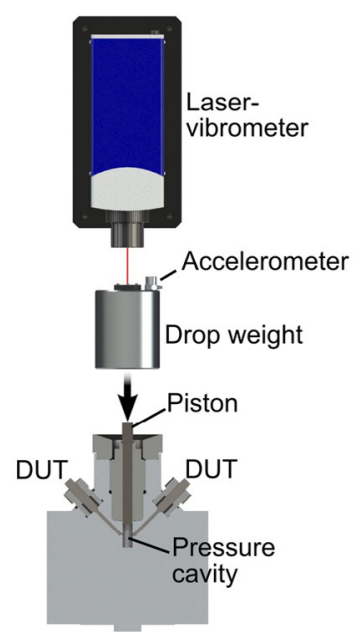

Fig. 8. Design of the MIKES pressure machine.

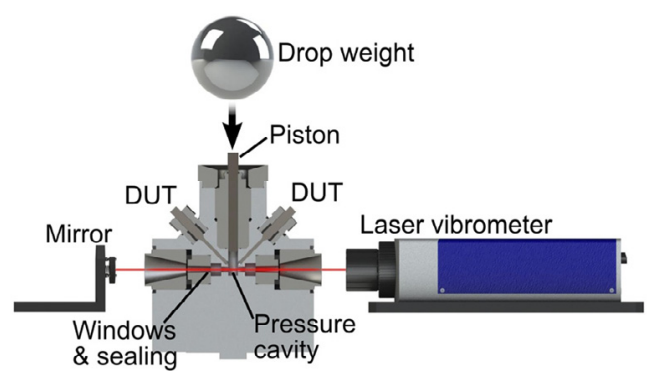

Fig. 9. Design of the PTB pressure machine.

during impact to deduce the force transmitted by the piston's cross-section into the hydraulic fluid. Thus via force and cross-section or via displacement and compressibility the actual pressure in the cavity can be determined. The first method gives rough estimations of pressure values, while the latter takes into account cavity and fluid effects, thus providing more information, improved traceability, and smaller uncertainties. The exact determination of the cavity volume, and changes in it due to piston movement, is the key requirement.

The PTB device is under modification to allow for an interferometric measurement of the optical path length through the pressure cavity, i.e., the hydraulic medium. This path length changes as the refractive index of the transmission medium varies as the fluid is compressed. In this approach the actual dynamic pressure can be linked to a static calibration of the optical measurement. The goal is to avoid any effects of inertia in the traceability chain between static and dynamic pressure. Preliminary tests of the measurement scheme are currently being performed in parallel to the modification of the drop weight device.

\subsection{Shock tube}

The dynamic pressure facilities of NPL operate according to another principle: the shock tube. They have developed a 1.4 MPa plastic shock tube with interchangeable driven sections of $2 \mathrm{~m}, 4 \mathrm{~m}$, and $6 \mathrm{~m}$ lengths. It includes a pressure transducer located in the centre of the end-wall. 

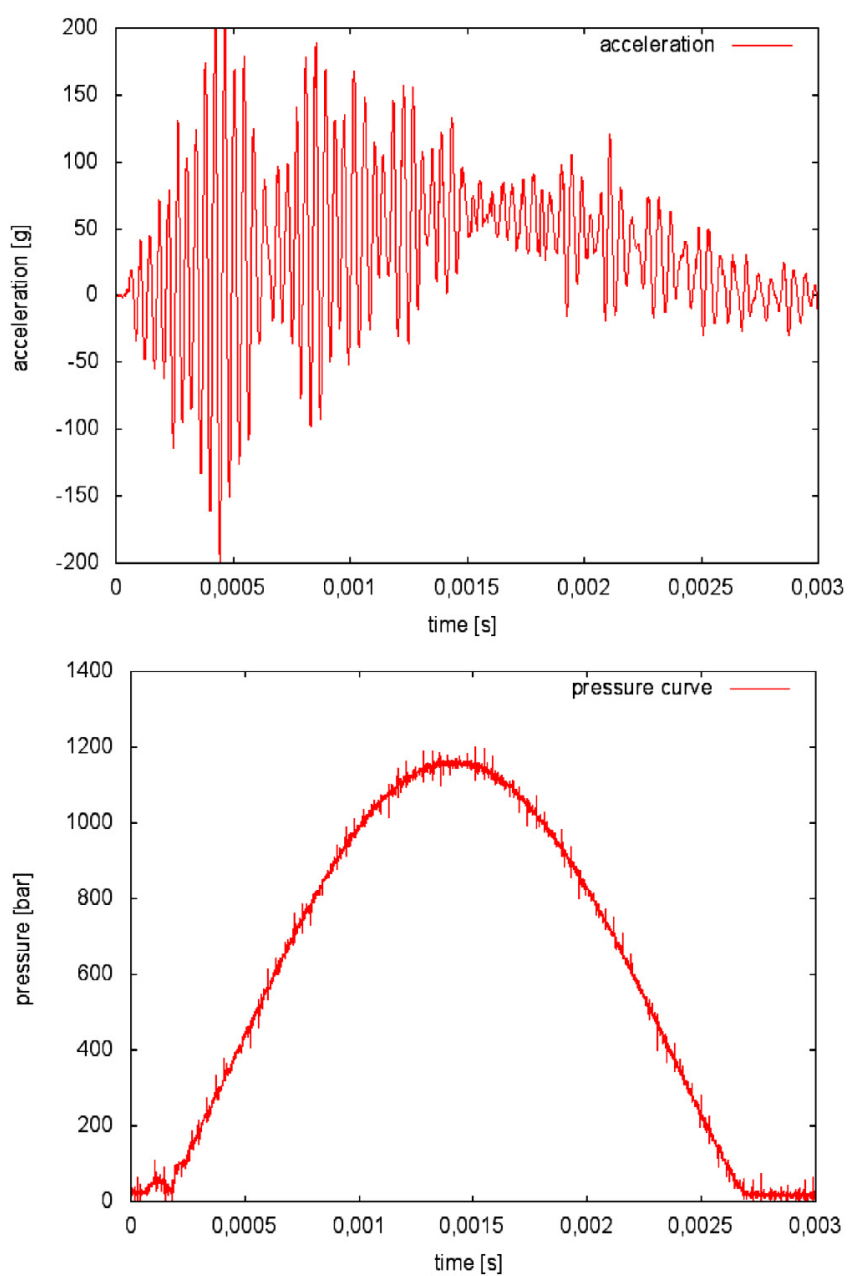

Fig. 10. Acceleration and pressure curves.

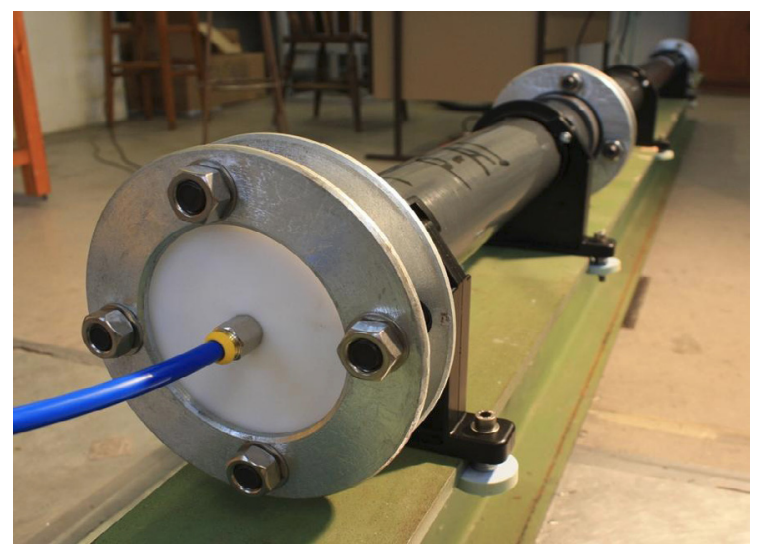

Fig. 11. Plastic shock tube of $0.7 \mathrm{~m}$ driver section and $2 \mathrm{~m}$ driven section.

It works by means of a pressurization system based on bottled nitrogen, using either single or double diaphragms. Future work will include shock wave velocity measurement (via side-wall mounted sensors) and end-wall acceleration measurement. The photographs in Figures 11 and 12 illustrate the shock tube and a diaphragm after the test.

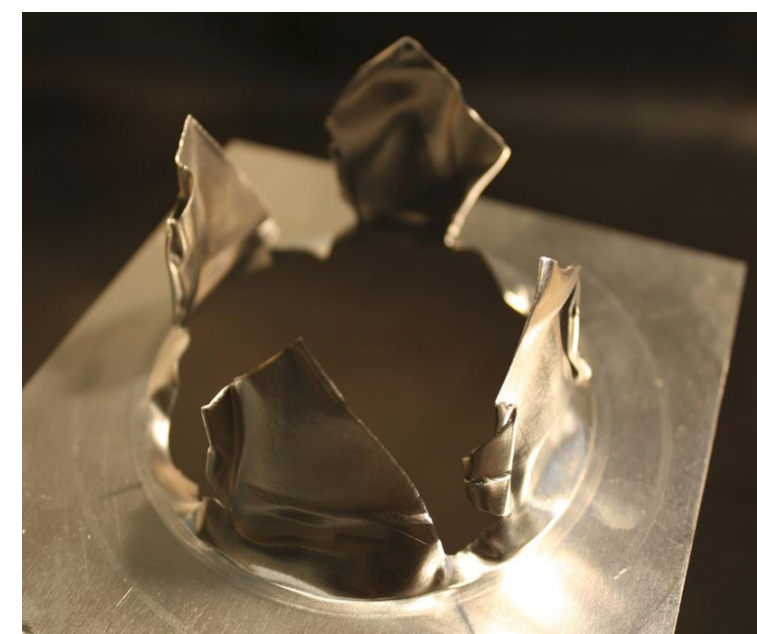

Fig. 12. Burst aluminum diaphragm.

NPL have begun the design of a $7 \mathrm{MPa}$ steel shock tube and plan to develop a second steel shock tube with a maximum pressure of $20 \mathrm{MPa}$.

A comparison of the different driven sections of the plastic tube, with different burst pressures and diaphragm arrangements, has been carried out. Results indicate that varying the burst pressure has the greatest influence on the characteristics of the end-wall pressure trace.

\section{WP 3: dynamic torque}

This work package is concerned with the development of methods and devices for dynamic torque measurements to provide a validated primary torque calibration for sinusoidal torque.

Three national metrology laboratories are involved in this work package: PTB, who is the work package leader, CMI (Czech Republic) and UME (Turkey). In addition, HBM (www.hbm.com), a leading German manufacturer of force and torque sensors, is taking part in the work as a collaboration partner.

PTB is currently the only participating NMI with research activities in this field of dynamic measurement. At PTB, an existing measurement set-up has been modified and improved to be able to provide dynamic torque calibrations of up to $20 \mathrm{~N} \cdot \mathrm{m}$ torque amplitude and up to $1 \mathrm{kHz}$ excitation frequency [9-11].

In the first months of this JRP, a new rotational exciter by Acutronic, Switzerland, was installed and commissioned (see Fig. 13). This exciter is able to achieve the required specifications and is equipped with an integrated rotational acceleration sensor. The rotational exciter is controlled by a state-of-the-art control system for sinusoidal excitation made by DataPhysics, USA.

In addition, the air bearing of the dynamic torque measuring device was replaced by a new air bearing of improved carrying capacity for parasitic radial loads and bending moments, as the performance of the old bearing suffered from wear possibly caused by parasitic loads.

The model description of the torque measuring device with the torque transducer under test requires the 


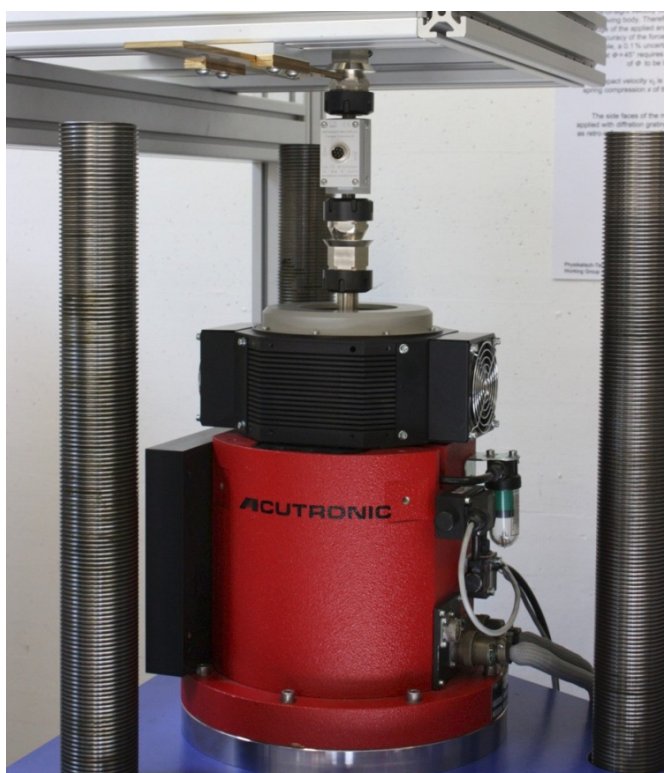

Fig. 13. Dynamic torque measuring device at PTB with the new rotational exciter.

parameter values of the coupled mechanical environment. To derive these parameters by experiment, two auxiliary measurement set-ups for the determination of torsional stiffness and moment of inertia have been designed and manufactured [11].

For the measurement of the torsional stiffness, in particular of the two couplings, PTB's static $20 \mathrm{~N} \cdot \mathrm{m}$ torque calibration machine was utilized to provide a defined torsional load. The resulting torsion angles are measured by means of two autocollimators directed at small mirrors attached to the tested structure.

The mass moment of inertia has to be determined for the couplings, the torque transducer under test, and the mechanical components coupled at the top of the dynamic torque measuring device (rotor of the air bearing, radial grating, coupling). For this purpose, a compound pendulum with airborne rotor that carries the device under test was designed and manufactured at PTB. The mechanical swing of the pendulum is measured by means of an optical angle measurement system. The oscillation frequency of the pendulum depends on the acting mass moment of inertia. By adding different auxiliary bodies of known mass moment of inertia, the wanted mass moment of inertia of the device under test can be calculated by extrapolation.

The next activities in WP 3 include the commissioning of the measuring devices for the determination of mass moment of inertia and torsional stiffness as well as the evaluation of the associated measurement uncertainties. Afterwards, the properties of the components of the dynamic torque calibration device will be determined, and the device will be assembled.

\section{WP 4: measuring amplifiers}

The aim of this work package is to establish a traceable dynamic calibration procedure for measuring amplifiers, as

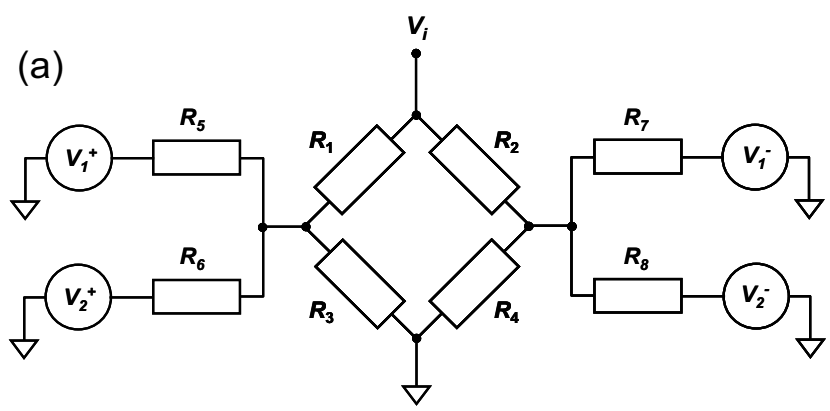

(b)

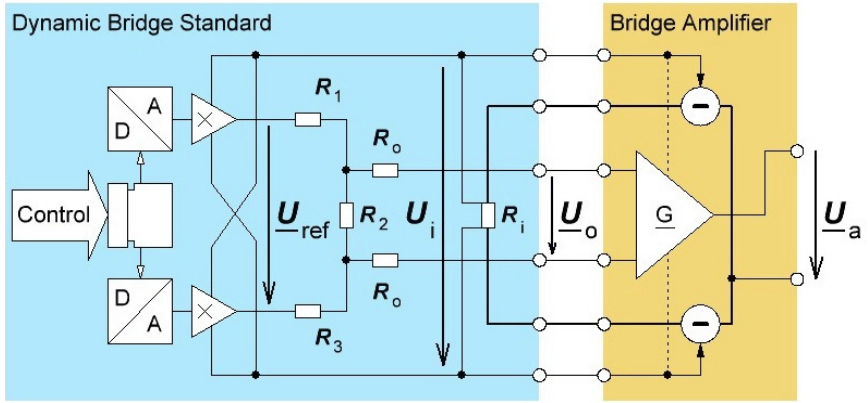

Fig. 14. Schematic of the strain gauge simulation in the NPL dynamic bridge standard (a) and operation principle of the PTB dynamic bridge standard (b).

part of the electric measurement chain for dynamic measurements of mechanical quantities in the frequency range from $\mathrm{DC}$ to $10 \mathrm{kHz}$.

In dynamic measurements two types of measuring amplifiers are commonly used, which are charge amplifiers for piezoelectric transducers and bridge amplifiers for strain gauge or piezoresistive transducers in Wheatstone bridge configuration.

In the case of charge amplifiers the calibration is less challenging, since the standard charge signal for calibration can be realized with help of a calibrated AC voltage source and a standard capacitor.

For bridge amplifiers the measurement task is more complex, since the measured transducer bridge output voltage $U_{0}$ is a ratiometric measurand in $\mathrm{mV} / \mathrm{V}$ and depends on the DC bridge supply voltage $U_{\mathrm{i}}$, which is provided by the bridge amplifier.

Consequently, the dynamic bridge standards need to work in the same ratiometric operation principle to simulate the strain gauge or piezoresistive transducers. Figure 14 shows the operation approaches of the NPL dynamic bridge standard (Fig. 14a) as described in [12] and the PTB dynamic bridge standard (Fig. 14b) [13]. Both dynamic bridge standards (DBS) generate the dynamic bridge output voltage with the help of digital-to-analogue converters (DAC). In case of the NPL DBS in Figure 14a the $\mathrm{AC}$ and $\mathrm{DC}$ components of the bridge output voltage are each generated by a separate $\mathrm{DAC}\left(V_{1}\right.$ and $\left.V_{2}\right)$ that uses the bridge supply voltage $V_{\mathrm{i}}$ as DAC reference voltage and adds up the signals with help of the attenuator resistors $\left(R_{5}\right.$ to $\left.R_{8}\right)$. 


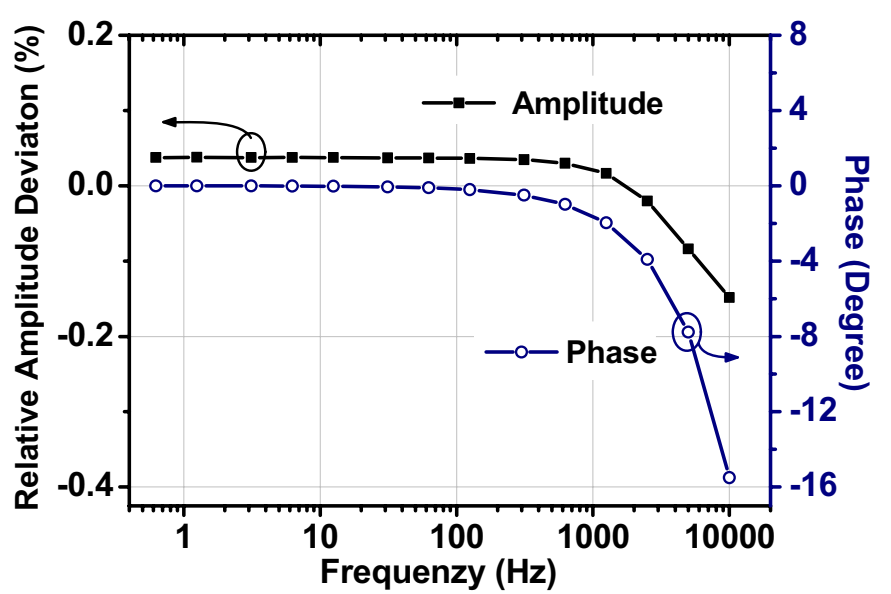

Fig. 15. Example measurement with the PTB dynamic bridge standard of the frequency dependent relative amplitude deviation and the phase of a typical strain gauge bridge amplifier in the $2 \mathrm{mV} / \mathrm{V}$ measurement range.

The PTB DBS in Figure 14b uses multiplying DACs (MDACs) to generate a static or dynamic signal $\left(U_{\text {ref }}\right)$, which is supplied to a resistive 1/200 voltage divider (resistors $R_{1}$ to $R_{3}$ ) with known amplitude and phase behavior to generate the bridge standard output signal $U_{0}$.

To match DBS impedances to the typical impedances of strain gauge transducers, the NPL DBS uses the bridge resistors $\left(R_{1}\right.$ to $\left.R_{4}\right)$ themselves and the PTB DBS uses the input resistor $\left(R_{i}\right)$ and the two output resistors $\left(R_{0}\right)$.

In the past month the NPL DBS prototype and PTB DBS prototype were modified and therefore extended in order to provide a sinusoidal reference signal, which is in-phase with the signal that simulates the strain gauge bridge voltage.

A preliminary frequency-dependent amplitude and phase characterization of a commercial bridge amplifier was carried out with help of the PTB DBS and is shown in Figure 15. The phase information was determined with the help of the MDAC signals, which are used as reference voltage $U_{\text {ref }}$, and the bridge amplifier output voltage amplitude $U_{\mathrm{a}}$. The measurements were carried out with a single synchronized sampling voltmeter, which is alternately sampling $U_{\text {ref }}$ and $U_{\mathrm{a}}$ with the help of a low resistive signal switch [14]. The results for amplitude and phase show characteristics similar to a low-pass filter with decreasing values beyond measurement frequencies of $1 \mathrm{kHz}$.

The next step in the project will be the traceable calibration of the DBS developed at NPL and PTB with respect to amplitude and phase.

\section{WP 5: mathematical and statistical methods and modelling}

The approach to modelling the new primary standard methodologies that are being developed during the course of the JRP is summarised below.

\subsection{Approach and underlying assumptions}

Our fundamental assumption is that all measurement systems that will be considered during the course of this JRP can be regarded as linear and time-invariant. This allows us to apply convolution and deconvolution methods and to regard the input-output characteristics of a system to be completely described by the system's impulse response.

Thus we expect that the measuring systems under consideration will be modelled by sets of linear ordinary differential equations, or by equivalent rational functions in the Laplace domain. We also expect that analysis will be performed mainly in the frequency domain.

To allow meaningful interpretation of calibration results, we intend as far as possible to develop parametric "white box" system models that take into account the known physical and engineering characteristics of the measuring system being developed during the course of the JRP, and to ensure traceability the methods set out in the Guide to the expression of uncertainty measurement [15] and its supplements will be applied. Any new methods that we develop will be in accordance with the underlying philosophy of the GUM and we intend that they can be viewed as implementing and extending the GUM methodology. Bayesian methods will be employed so as to allow prior knowledge obtained either from experiments or from experts to be incorporated into the uncertainty evaluation process.

\subsection{Relevant prior work by the WP 5 team}

The methods being developed rely on important prior work by members of the WP5 team, specifically:

- prior work on analysis of dynamic measurements by PTB [16-19] and in particular their work on the implementation of uncertainty evaluation methods based on the Guide to the expression of uncertainty in measurement;

- prior work on dynamic calibration of accelerometers and force transducers by PTB, including analysis of sinusoidal and shock excitation measurements and analysis of comparison measurements between different laboratories [20-25];

- joint work by mathematicians at PTB and NPL to study dynamic pressure measurements carried out by NPL experimentalists using a shock tube that is located at the UK's Cranfield University [26];

- joint work by PTB, NPL and the SP Technical Research Institute of Sweden on deconvolution methods for the analysis of dynamic measurements [27];

- joint work by PTB and NPL on the implementation of a GUM Monte Carlo method for dynamic measurements [28].

\subsection{Example modelling for the measurand dynamic torque}

In this section, the general approach of a model-based analysis is exemplarily presented for the measurement of 


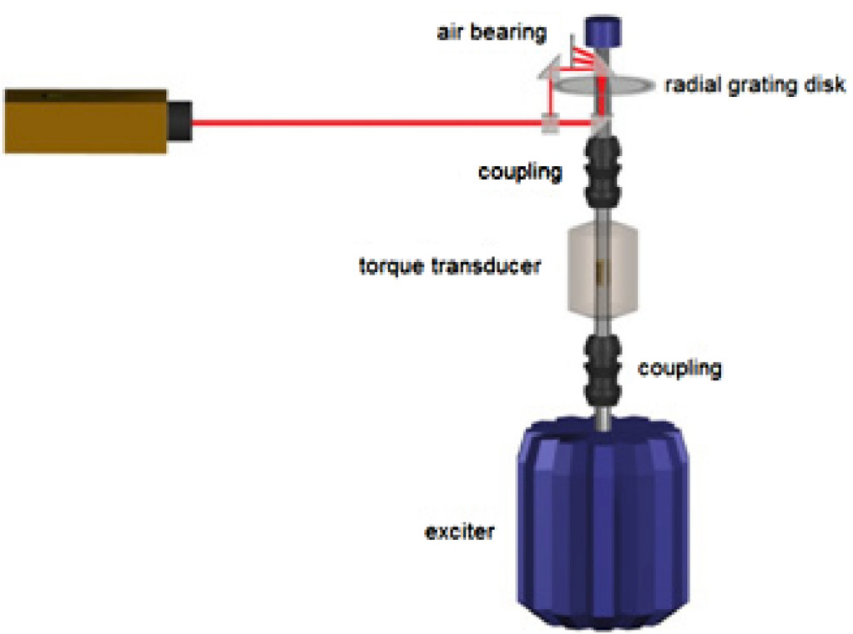

Fig. 16. Schematic representation of a dynamic torque calibration device [10].

dynamic torque. The model will describe the dynamic behaviour of the dynamic measuring device at PTB, which was introduced in the previous section of WP 3 .

Figure 16 shows a schematic picture of this device with the main components rotational exciter, couplings, torque transducer under test, and interferometric measurement of angular acceleration.

The signal of the torque transducer will undergo conditioning before being captured by the data acquisition system. Both the signal conditioning system and the data acquisition system must be taken into account during data analysis. Information about the amplifier will take the form of calibrated frequency response values at the relevant frequencies. In addition to the torque transducer output, angular acceleration at the top of the calibration device will be measured using a traceable calibrated rotational vibrometer. After multiplication by an appropriate mass moment of inertia, this angular acceleration gives rise to a reference torque signal. Angular acceleration at the bottom of the device will be measured using a traceable calibrated sensor built in the exciter for this purpose. Data will be provided in the frequency domain, i.e., values of the frequency response functions (linking the output of the transducer with acceleration as input) will be available in a straightforward manner.

This calibration device will be modelled as a series of three torsional spring-damper systems (one for each coupling and one for the transducer), see Figure 17 [11]. The aim of the measurement scheme is to identify the parameters of the model for the transducer, namely the torsional stiffness, damping, mass moments of inertia at the head and base of the transducer, and a proportionality parameter. The spring-damper system is described by an appropriate set of differential equations that can be employed in the evaluation of the model parameters of interest.

\section{Conclusion}

With the funding of the joint research Project IND09, the European Metrology Research Programme and the

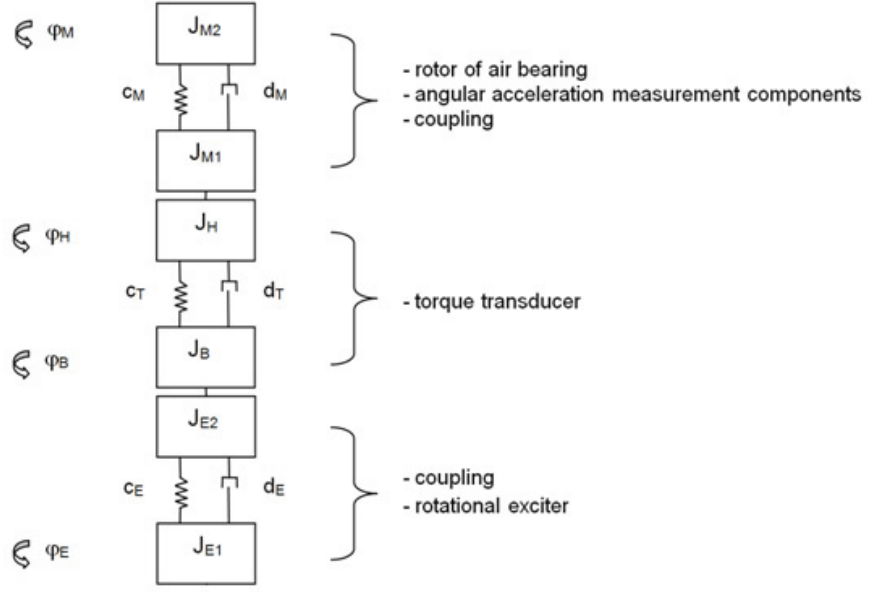

Fig. 17. Model of the dynamic calibration device for torque transducers [11].

European Commission provide a unique opportunity for a group of European NMIs to develop a new field of metrology. The aim of the JRP is the development of a basic infrastructure in terms of devices and methods to provide traceability for dynamic measurements of the mechanical quantities force, torque, and pressure.

Facilities are being developed for the three mechanical quantities and for the measuring amplifiers. Data will be soon available as input to the mathematics work package.

The immediate next steps for each technical work package are:

- WP 1: to continue the characterization of the facilities for the three participating laboratories including the determination of the uncertainties components.

- WP 2: to continue development of primary dynamic pressure generation systems at the four participating NMIs and then to carry out a set of comparisons between them.

- WP 3: to commission the measuring devices for the determination of the mass moment of inertia and torsional stiffness and to evaluate their uncertainties.

- WP 4: to develop a traceable calibration of the dynamic bridge standards (DBS) at both contributing NMIs (PTB and NPL) and to provide the DBS for the dynamic calibration of strain gauge bridge amplifiers.

- WP 5: to begin developing modelling and uncertainty analysis software that can be used to support NMIlevel primary standard calibrations for the three mechanical quantities of interest.

Acknowledgements. The research leading to these results has received funding from the European Union on the basis of Decision No. 912/2009/EC.

\section{References}

1. EMRP outline 2008 document

2. C. Schlegel, G. Kiekenap, B. Glöckner, R. Kumme, Dynamic calibration of force sensors using sinusoidal excitations, Sensordevices 2011, Nice, France 
3. C. Schlegel, G. Kiekenap, B. Glöckner, A. Buß, R. Kumme, Traceable periodic force calibration, Metrologia 49, 224235 (2012)

4. M. Kobusch, T. Bruns, The new impact force machine at PTB, in Proc. of XVII IMEKO World Congress, Dubrovnik, Croatia, 2003, pp. 263-267

5. M. Kobusch, T. Bruns, Uncertainty contributions of the impact force machine at PTB, in Proc. of XVIII IMEKO World Congress, Rio de Janeiro, Brazil (CD publication, 2006)

6. M. Kobusch, T. Bruns, L. Klaus, M. Müller, The $250 \mathrm{kN}$ primary shock force calibration device at PTB, in Measurement, Special Issue: IMEKO 2010, Pattaya, Thailand (Elsevier), in press

7. M. Kobusch, L. Klaus, T. Bruns, Model-based analysis of the dynamic behaviour of a $250 \mathrm{kN}$ shock force calibration device, in XX IMEKO World Congress, Busan, Republic of Korea, 2012, to be published

8. M. Kobusch, T. Bruns, E. Franke, Challenges in practical dynamic calibration, in Advanced Mathematical and Computational Tools in Metrology and Testing (World Scientific, 2009), pp. 204-212

9. T. Bruns, Sinusoidal torque calibration: A design for traceability in dynamic torque calibrations, in XVII IMEKO World Congress, Dubrovnik, Croatia, 2003, pp. 282-285

10. T. Bruns, M. Kobusch, Traceability of dynamic force and torque calibrations by means of laser-Dopplerinterferometry, Proc. SPIE 5503, 602-607 (2004)

11. L. Klaus, T. Bruns, M. Kobusch, Determination of model parameters for a dynamic torque calibration device, in $X X$ IMEKO World Congress, Busan, Republic of Korea, 2012, to be published

12. J.M. Williams, D.R. Smith, D. Georgakopoulos, P.D. Patel, J.R. Pickering, Design and metrological applications of a low noise, high electrical isolation measurement unit, IET Sci. Meas. Technol. 3, 165-174 (2009)

13. M.F. Beug, H. Moser, G. Ramm, Dynamic bridge standard for strain gauge bridge amplifier calibration, Conference on Precision Electromagnetic Measurements (CPEM), 2009

14. G. Ramm, H. Moser, A. Braun, A new scheme for generating and measuring active, reactive, and apparent power at power frequencies with uncertainties of $2.5 \times 10^{-6}$, IEEE Trans. Instrum. Meas. 48, 422-426 (1999)

15. BIPM, IEC, IFCC, ILAC, ISO, IUPAC, IUPAP, and OIML, Evaluation of measurement data - Guide to the expression of uncertainty in measurement. Joint Committee for Guides in Metrology, JCGM 100:2008

16. S. Eichstädt, Analysis of dynamic measurements Evaluation of dynamic measurement uncertainty, Ph.D. thesis, PTB report IT-16 (2012)
17. S. Eichstädt, A. Link, C. Elster, Dynamic uncertainty for compensated second-order systems, Sensors 10, 7621-7631 (2010)

18. A. Link, C. Elster, Uncertainty evaluation for IIR (infinite impulse response) filtering using a state-space approach, Meas. Sci. Technol. 20, 055104 (2009)

19. C. Elster, A. Link, Uncertainty evaluation for dynamic measurements modelled by a linear time-invariant system, Metrologia 45, 464-473 (2008)

20. S. Eichstädt, A. Link, T. Bruns, C. Elster, Online dynamic error compensation of accelerometers by uncertainty-optimal filtering, Measurement 43, 708-713 (2010)

21. G. Wübbeler, A. Link, T. Bruns, C. Elster, Impact of correlation in the measured frequency response on the results of a dynamic calibration, in Advanced mathematical \& computational tools in metrology VIII, Series on Advances in Mathematics for Applied Sciences, edited by F. Pavese, M. Bär, J.M. Limares, C. Perruchet, N.F. Zhang (World Scientific, New Jersey, 2009), Vol. 78, pp. 369-374

22. C. Elster, A. Link, T. Bruns, Analysis of dynamic measurements and determination of time-dependent measurement uncertainty using a second-order model, Meas. Sci. Technol. 18, 3682-3687 (2007)

23. A. Link, A. Täubner, W. Wabinski, T. Bruns, C. Elster, Modelling accelerometers for transient signals using calibration measurements upon sinusoidal excitation, Measurement 40, 928-935 (2007)

24. A. Link, A. Täubner, W. Wabinski, T. Bruns, C. Elster, Calibration of accelerometers: determination of amplitude and phase response upon shock excitation, Meas. Sci. Technol. 17, 1888-1894 (2006)

25. M. Kobusch, A. Link, A. Buss, T. Bruns, Comparison of shock and sine force calibration methods, in Proc. of IMEKO TC3 \& TC 16 \& TC22 International Conference, Merida, Mexico, 2007

26. T. Esward, C. Matthews, S. Downes, A. Knott, S. Eichstädt, C. Elster, Uncertainty evaluation for traceable dynamic measurement of mechanical quantities: a case study in dynamic pressure calibration, in Advanced mathematical \&3 computational tools in metrology IX, Göteborg, Sweden, 2011, in press

27. S. Eichstädt, C. Elster, T.J. Esward, J.P. Hessling, Deconvolution filters for the analysis of dynamic measurement processes: a tutorial, Metrologia 47, 522-533 (2010)

28. S. Eichstädt, A. Link, P. Harris, C. Elster, Efficient implementation of a Monte Carlo method for uncertainty evaluation in dynamic measurements, Metrologia 49, 401-410 (2012) 